\title{
The Thromboplastic Activity of Lung Surfactant in Amniotic Fluid and Its Application to Prenatal Assessment of Fetal Lung Maturity
}

\author{
Motokazu Higuchi, Hideto Hirano and Masahiro Maki \\ Department of Obstetrics and Gynecology, Akita University \\ School of Medicine, Akita 010
}

\begin{abstract}
Higuchi, M., Hirano, H. and Maki, M. The Thromboplastic Activity of Lung Surfactant in Amniotic Fluid and Its Application to Prenatal Assessment of Fetal Lung Maturity. Tohoku J. exp. Med., 1981, 133 (3), 267-273 — Based on the fact that both tissue thromboplastin and lung surfactant show lamellar structures under the electron microscope and belong chemically to lipoprotein, the thromboplastic activity of lung surfactant in amniotic fluid was studied by measuring plasma recalcification time. The results obtained were as follows: (1) The surfactant fractions isolated from amniotic fluid and rabbit or pig lung showed the thromboplastic activity with dose response. (2) The thromboplastic activity of amniotic fluid increased with advancing gestational age. (3) It was found that the thromboplastic activity determined by plasma recalcification time was parallel with the surfactant concentration of amniotic fluid. (4) The shortening rate of plasma recalcification time in amniotic fluid could estimate well the risk of RDS, and the critical value for RDS was assumed to be about $33 \%$. lung surfactant; amniotic fluid; thromboplastin; fetal lung maturation
\end{abstract}

The development of respiratory distress syndrome (RDS) can be predicted by various chemical determinations of surfactant components, for example, total lecithin (Bhagwanani et al. 1972) or lecithin/sphingomyelin ratio (Gluck et al. 1971). However, the procedures are relatively complicated and time consuming to perform. Simple and reliable guide information of surfactant concentration in amniotic fluid can be obtained by the shake test (Clements et al. 1972) and more precisely by its modification, that is, the double repeat shake test (Higuchi et al. 1978). In this study, another simple method will be discussed.

Since both lung surfactant and tissue thromboplastin show lamellar structures under the electron microscope (Hasegawa et al. 1977) and belong chemically to lipoprotein (Abrams and Taylar 1964; Galdston et al. 1969), the thromboplastic activity of surfactant preparations from lung and amniotic fluid was studied by measuring plasma recalcification time (PRCT). After confirmation of the thromboplastic activity of the lung surfactant, we studied whether the concentration of lung surfactant in amniotic fluid could be simply measured by employing the clot promoting activity of surfactant.

Received for publication, February 23, 1980. 


\section{Materials and Methods}

\section{Isolation of surfactant from lung and amniotic fluid}

Surfactant was extracted from pig and rabbit lungs by washing out to and from with $0.145 \mathrm{M}$ saline solution $(0.54 \mathrm{ml} / \mathrm{g}$ tissue). The washing was centrifuged at $200 \times \mathrm{g}$ for $20 \mathrm{~min}$ to remove cells and particulates. Surfacant in the supernatant was further purified by a modification of Frosolono's method (Frosolono et al. 1970) as shown in Fig. 1.

Amniotic fluid was obtained from pregnant women at 25 weeks to 41 weeks by therapeutic amniocentesis. Surfactant in amniotic fluid was isolated by the same way as above. In the final step, we obtained the centrifuge pattern as shown in Fig. 1, and only the interface 3 had surfactant activity. The surfactant fraction was suspended in 0.145 $\mathrm{M}$ saline solution, and its concentration was expressed as $\mathrm{mg} / 100 \mathrm{ml}$ of phospholipid after determining phosphorus.

\section{Assay of thromboplastic activity of surfactant}

The thromboplastic activity of the surfactant fractions was measured by plasma recalcification time (PRCT). A mixture of each $0.1 \mathrm{ml}$ standard normal plasma (Dade, AHSC, U.S.A.) and surfactant fraction or $0.145 \mathrm{M}$ saline solution as control was incubated at $37^{\circ} \mathrm{C}$ for $10 \mathrm{~min}$. After the incubation, $0.1 \mathrm{ml}$ of $0.02 \mathrm{M} \mathrm{CaCl}_{2}$ was added and the clotting time was recorded. The shortening rate of PRCT was calculated using the formula of (control PRCT-test PRCT)/control PRCT $\times 100(\%)$.

\section{Assay of surfactant activity in amniotic fluid by shake test}

A modification of the shake test by Clements et al. (1972) was employed to measure more precisely the surfactant activity (Higuchi et al. 1978).

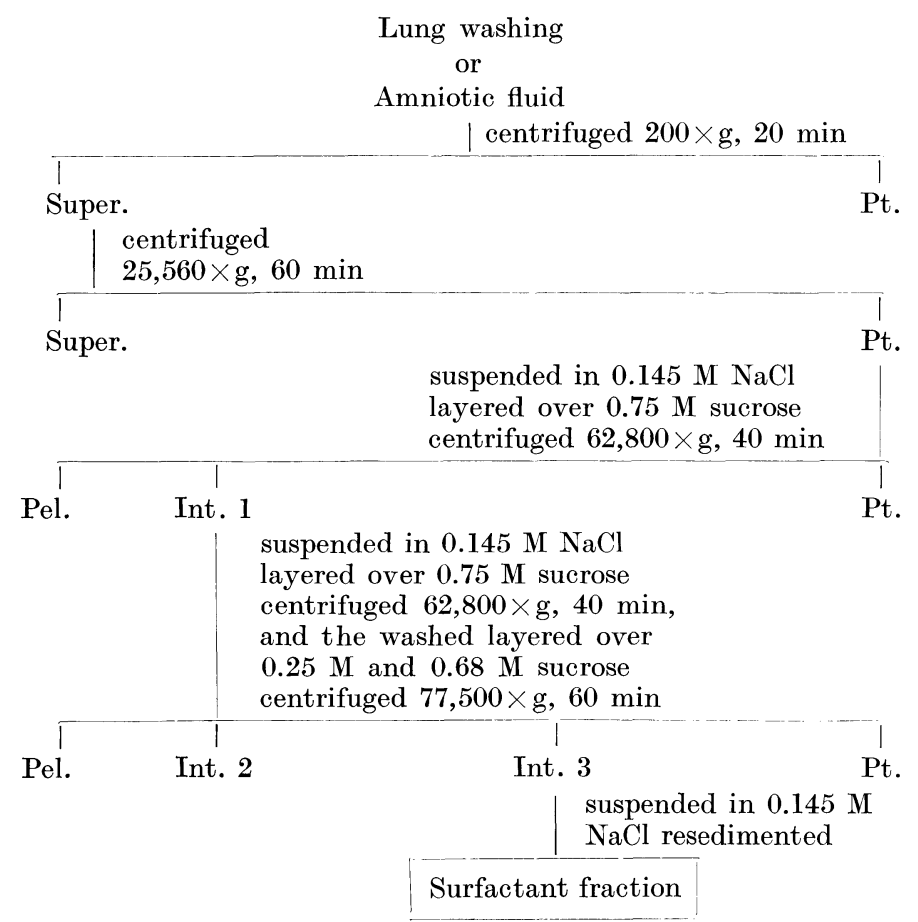

Fig. 1. Isolation of surfactant from lung washing or amniotic fiuid.

Super., supernatant; Pt., precipitate; Pel., pellicle; Int., interface. 


\section{Results}

\section{Thromboplastic activity of lung surfactant}

The shortening rate of PRCT is shown in Fig. 2. It was clearly demonstrated that the surfactant fraction isolated from rabbit and pig lung had the clot promoting activity with dose response in a certain range. Dipalmitoyl lecithin (DPL: SIGMA, U.S.A.), a major component of lung surfactant phospholipid, however, did not exert this effect.

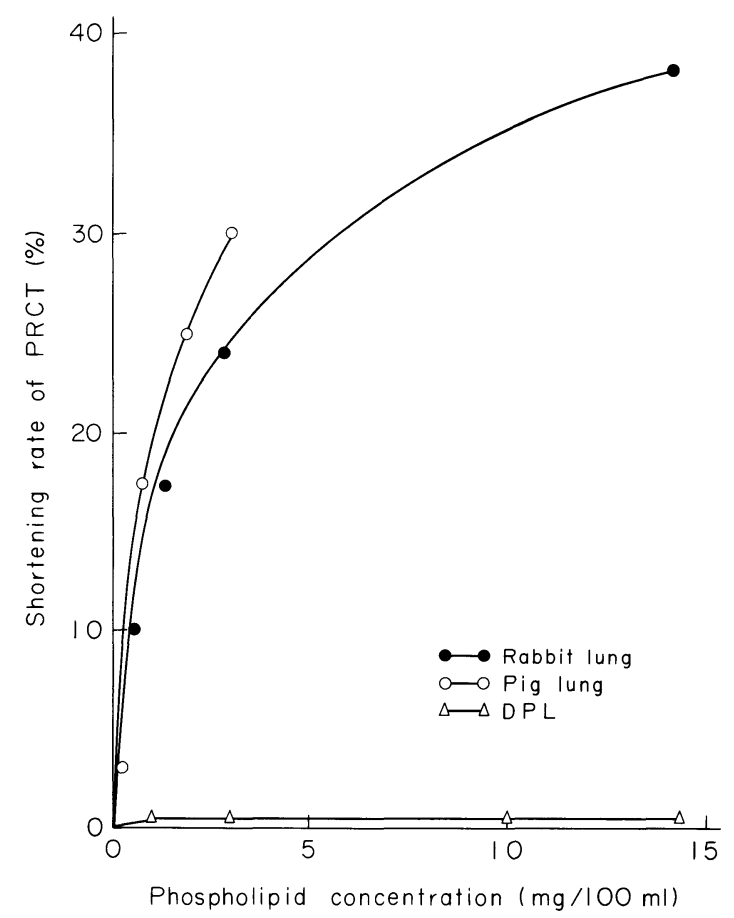

Fig. 2. Lung surfactant and shortening rate $(\%)$ of PRCT.

\section{Thromboplastic activity of surfactant fraction}

As shown in Fig. 3, surfactant fraction isolated from amniotic fluid showed the shortening activity of PRCT. Amniotic fluid, after removing the surfactant by centrifugation at $25,560 \times \mathrm{g}$ for $60 \mathrm{~min}$, reduced markedly its activity. Also, the sediment of amniotic fluid at $1,000 \times \mathrm{g}$ for 20 min showed the shortening activity of PRCT.

Application of the amniotic fluid PRCT to prenatal assessment of fetal lung maturation

In order to apply the PRCT to prediction of fetal lung maturity, the PRCT was measured on 5-times diluted supernatant of amniotic fluid after centrifugation at $1,000 \times \mathrm{g}$ for $20 \mathrm{~min}$. 


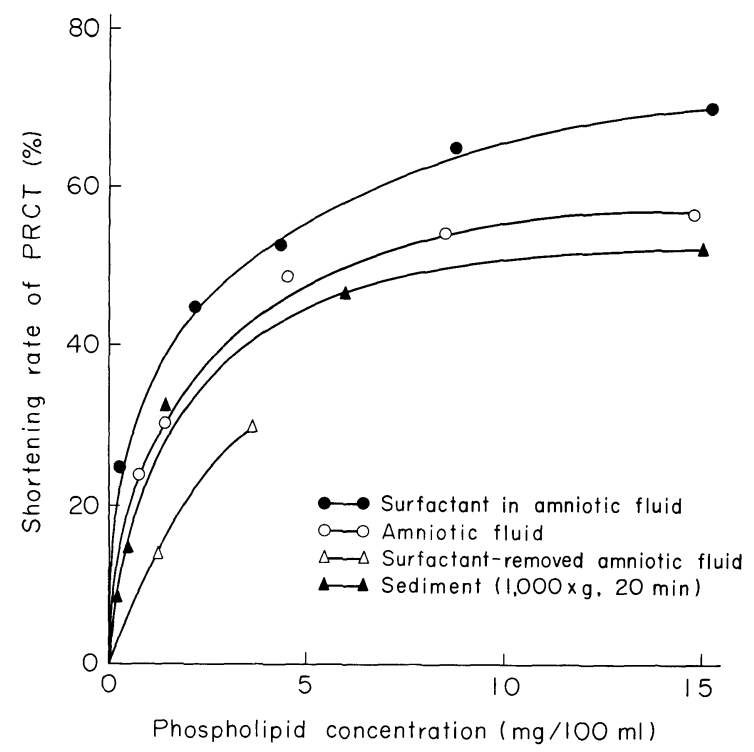

Fig. 3. Surfactant in amniotic fluid and shortening rate (\%) of PRCT.

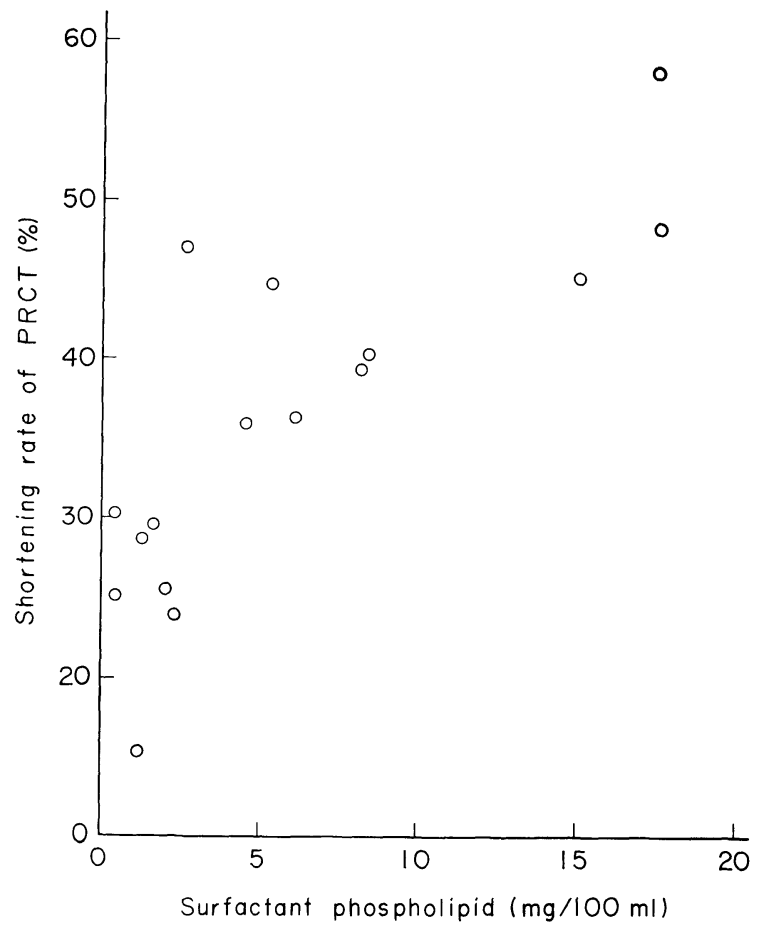

Fig. 4. Correlation between shortening rate $(\%)$ of PRCT and surfactant phospholipid in amniotic fluid. 


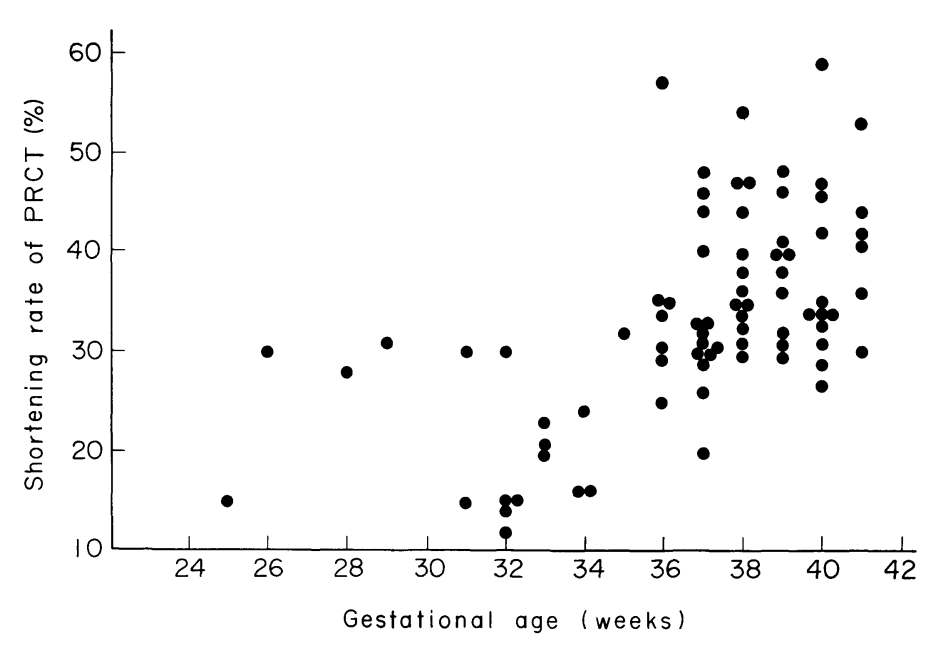

Fig. 5. Shortening rate $(\%)$ of PRCT and gestational age.

Simultaneous measurements of PRCT and surfactant phospholipid concentrations were done on 16 amniotic fluid samples at various stages of gestation. The results are shown Fig. 4. A close correlation was observed between them $(r=0,77)$. In all of 4 cases in which the concentrations of surfactant phospholipid were less than $1.0 \mathrm{mg} / 100 \mathrm{ml}$, the critical value for RDS, the shortening rates of PRCT were less than $35 \%$. In 10 of 12 cases in which the concentrations of surfactant phospholipid were more than $1.0 \mathrm{mg} / 100 \mathrm{ml}$, the shortening rates of PRCT were more than $35 \%$. From these results, amniotic fluid PRCT was suggested to reflect well surfactant concentration in amniotic fluid.

Correlation between thromboplastic activity of amniotic fluid and gestational age

The shortening rates of PRCT were $30 \%$ or less until 35 weeks of gestation and increased rapidly to more than $30 \%$ in 39 of 41 cases on and after 38 weeks gestation (Fig. 5).

\section{Correlation between thromboplastic activity of amniotic fluid and surfactant titer}

Samples of amniotic fluid used here were obtained from 64 pregnant women within $24 \mathrm{hr}$ before delivery. As shown in Fig. 6, it was clear that there was a close correlation between shortening rate of PRCT and surfactant titer by the shake test. When surfactant titers by the shake test were judged as clearly negative (negative in original undiluted amniotic fluid) or as intermediate (positive in undiluted amniotic fluid, but negative in 1:2 dilution), the shortening rates of PRCT were less than $33 \%$ except for one case. In all of 5 RDS cases the shortening rates of PRCT were less than $33 \%$. In most of cases in which surfactant titers were clearly positive (positive in 1:2 dilution or more), the shortening rates of PRCT were more than $33 \%$ and, furthermore, no infant developed RDS. 


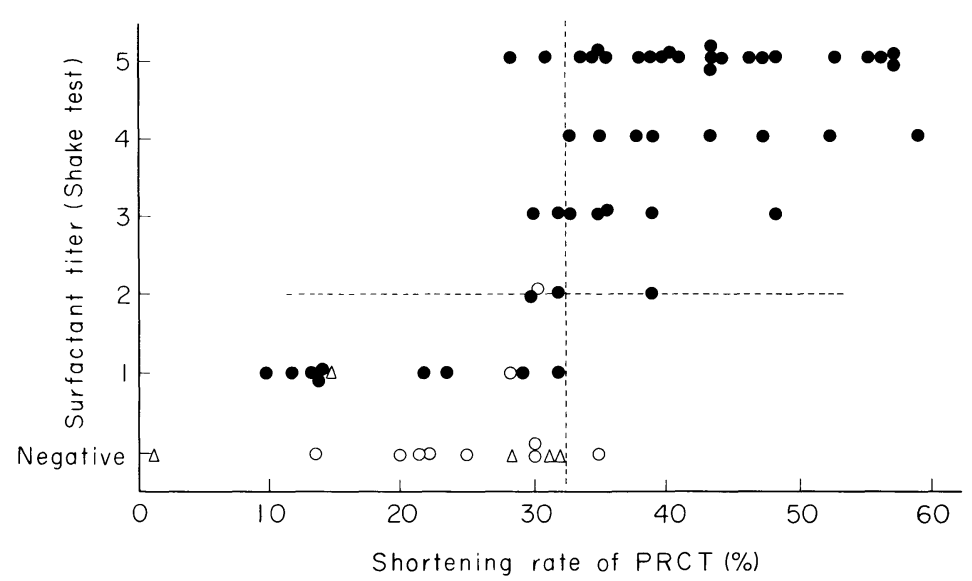

Fig. 6. Correlation between surfactant titer and shortening rate (\%) of PRCT. $\bullet$, at delivery, no RDS; o, not delivered; $\Delta$, RDS.

\section{Discussion}

It is widely accepted that lung surfactant plays an important role in stabilizing alveolar air spaces by forming the alveolar lining layer after birth. The fetal lung surfactant is excreted through fetal trachea into amniotic fluid with advancing fetal lung maturation.

Fujiwara and Higuchi (1973) isolated surfactant from human amniotic fluid by a modification of Frosolono's method of discontinuous sucrose density gradient centrifugation. This surfactant fulfiled the established criteria for lung surfactant; namely, phospholipid-protein ratio was 6.6, the major component of phospholipid was lecithin, in which dipalmitoyl lecithin was about 85 percent, and its minimum surface tension was extremely low as in lung surfactant. This surfactant concentration is a good indication of lung maturation; however, this method is too complicated and time consuming for clinical use. The determination of surfactant in amniotic fluid is often required to be done simply and rapidly.

There are three possible assay methods to determine the surfactant in amniotic fluid. They are (1) chemical assay of total lecithin or lecithin/sphingomyelin ratio which already reported by several investigators, (2) physicochemical assay of surfactant by the shake test, and (3) the clotting method which we studied in this paper.

As mentioned before, both lung surfactant and tissue thromboplastin show lamellar structures and belong chemically to lipoprotein; therefore, the thromboplastic activity of surfactant preparations from the lung and amniotic fluid was studied by measuring PRCT.

It was proved that both lung surfactant and surfactant fraction isolated from amniotic fluid had the thromboplastic activity. Furthermore, we attempted to develop the method for diagnosis of fetal lung maturation employing this character of surfactant, and obtained almost satisfactory results.

Hastwell (1978) reported that the thromboplastic activity in amniotic fluid 
reflected well the overall fetal maturity, and Suzuki et al. (1976) described that its activity has correlation with surfactant titer by shake test. Moreover, Yaffe et al. (1977) demonstrated that the thromboplastic activity of amniotic fluid was correlated with the lecithin-sphingomyelin ratio and with the clinical outcome of the newborn. Their findings are supported by the present study that the thromboplastic activity in amniotic fluid is mainly due to surfactant lipoprotein. The shortening rate of PRCT in amniotic fluid can estimate well the risk of RDS, and the critical value for RDS is assumed to be $33 \%$. Therefore, this method is considered to have a great value in predicting the neonatal respiratory function. The diagnostic accuracy will be higher when combined with shake test. The amniotic fluid PRCT can be determined simply and rapidly as routine clinical examination without using any special apparatus.

\section{References}

1) Abrams, M.E. \& Taylar, F.B. (1964) Isolation and quantitative estimation of pulmonary surface active lipoprotein and its interaction with fibrinogen. Physiologist, 7, 78.

2) Bhagwanani, S.G., Fahmy, D. \& Turnbull, A.C. (1972) Prediction of neonatal respiratory distress by estimation of amniotic-fluid lecithin. Lancet, 1, 159-162.

3) Clements, J.A., Platzker, A.C.G., Tierney, D.F., Hobel, C.J., Creasy, R.K., Margolis, A.J., Thibeault, D.W., Tooley, W.H. \& Oh, W. (1972) Assessment of the risk of the respiratory-distress syndrome by a rapid test for surfactant in amniotic fluid. New Engl. J. Med., 286, 1077-1081.

4) Frosolono, M.F., Charms, B.L., Pawlowski, R. \& Slivka, S. (1970) Isolation, characterization, and surface chemistry of surface-active fraction from dog lung. J. Lipid Res., 11, 439-457.

5) Fujiwara, T. \& Higuchi, M. (1973) Isolation, purification and quantitation of a surface-active material from human amniotic fluid: Correlation with gestational age and the surfactant titer. Proc. Jap. Soc. Biol. Interface, 4, 200-206.

6) Galdston, M., Shah, D.O. \& Shinowara, G.Y. (1969) Isolation and characterization of a lung lipoprotein surfactant. J. Colloid Interface Sci., 29, 319-334.

7) Gluck, L., Kulovich, M.V., Borer, R.C., Brenner, P.H., Anderson, G.G. \& Spellacy, W.N. (1971) Diagnosis of the respiratory distress syndrome by amniocentesis. Amer. J. Obstet. Gynecol., 109, 440-445.

8) Hasegawa, H., Nagata, H. \& Murano, M. (1977) Studies on the tissue thromboplastin during the coagulation-fibrinolytic process-ultrastructural changes. Thrombos. Haemostas., 37, 541-548.

9) Hastwell, G.B. (1978) Accelerated clotting time: An amniotic fluid thromboplastic activity index of fetal maturity. Amer. J. Obstet. Gynecol., 131, 650-654.

10) Higuchi, M., Soga, K., Hirano, H. \& Maki, M. (1978) On 342 amniotic fluid samples, a comparison of the surfactant lipoprotein and three simple and rapid testes for prediction of fetal lung maturity. Acta obstet. gynaec. jpn., 30, 1598.

11) Suzuki, S., Wake, N. \& Yoshiaki, K. (1976) New neonatal problems of blood coagulation and fibrinolysis II. Thromboplastic effect of amniotic fluid and its relation to lung maturity. J. Perinat. Med., 4, 221-226.

12) Yaffe, H., Bar-On, H., Eldor, A., Ron, M. \& Sadovsky, E. (1977) Correlation between thromboplastic activity and lecithin/sphingomyelin ratio in amniotic fluid. Preliminary report. Brit. J. Obstet. Gynaecol., 84, 354-356. 